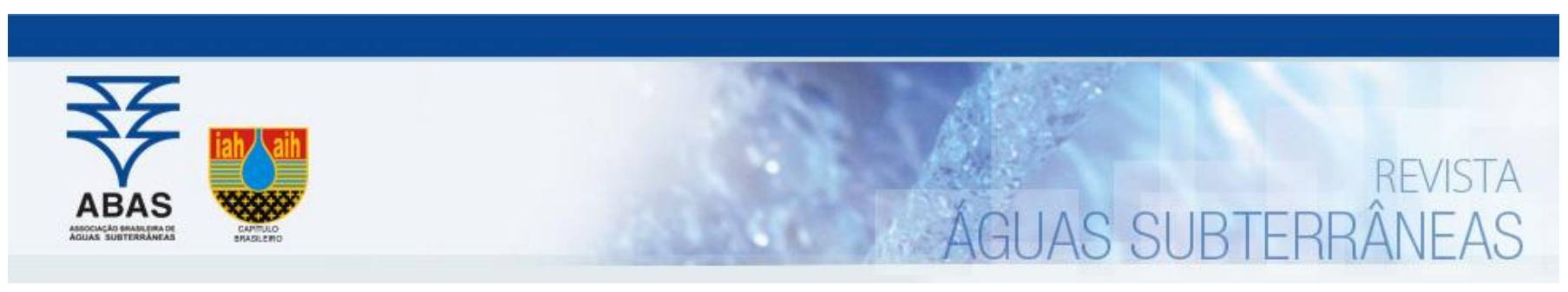

Artigos

\title{
Trapeamento de LNAPL observado por meio da técnica de fluorescência induzida por laser (LIF)
}

\section{LNAPL trapping observed by Laser-Induced Fluorescence (LIF) technique}

\author{
Elias Isler1,2; Elias Hideo Teramoto3,5; Marcus Paulus Martins Baessa4; Marco Aurélio Zequim Pede ${ }^{6}$; Chang Hung Kiang3,5,7凶 \\ 1 Universidade Federal do Oeste da Bahia - CCET / UFOB \\ 2 Programa de Pós-Graduação em Geociências e Meio Ambiente, UNESP - Campus de Rio Claro/SP \\ 3 Laboratório de Estudos de Bacias, UNESP - Campus de Rio Claro/SP \\ ${ }_{4}$ Centro de Pesquisas Leopoldo Américo Miguez de Mello - Petrobras \\ ${ }^{5}$ Centro de Estudos Ambientais, UNESP - Campus de Rio Claro/SP \\ 6 In-Situ Remediation \\ 7 Departamento de Geologia Aplicada, UNESP - Campus de Rio Claro/SP
}

\elias.isler@ufob.edu.br, teramoto@rc.unesp.br, marcus.baessa@petrobras.com.br, mpede@yahoo.com, chang@rc.unesp.br

\begin{abstract}
Resumo
Palavras-chave:

Oscilação do NA.

Smear Zone.

Querosene de Aviação.

Atenuação Natural.

Modelos conceituais de áreas contaminadas são, em geral, os primeiros resultados significativos do gerenciamento ambiental. Contudo, é necessário entender a complexidade dos meios contaminados para que seja adotada a melhor alternativa de remediação. A distribuição espacial em subsuperfície da fase liquida não aquosa menos densa que a água (LNAPL - Light Non-Aqueous Phase Liquid), geralmente de elevada variabilidade espacial e, consequentemente, complexa, deve ser mais bem conhecida. Além disso, a flutuação sazonal do nível d'água promove os fenômenos de trapeamento e destrapeamento de LNAPL e define uma zona de redistribuição vertical (smear zone) da contaminação, aumentando a complexidade do cenário. Com o objetivo de preencher lacunas deixadas pelas técnicas convencionais de investigação, foi empregada a técnica de fluorescência induzida por laser (LIF) para avaliação do fenômeno de trapeamento em uma área contaminada por querosene de aviação, situada no interior do estado de São Paulo. Os resultados dos ensaios de fluorescência e medição do nível d'água do aquífero local indicam LNAPL trapeado na porção saturada com limites bem marcados que definem a smear zone. Análises qualitativas revelam similaridade da contaminação na maioria dos pontos investigados, com baixa intensidade de sinal de fluorescência nos menores comprimentos de onda de resposta, compatível com depleção de hidrocarbonetos mais leves pela atenuação natural.
\end{abstract}

Keywords:

Water-level Fluctuation.

Smear Zone.

Kerosene-type Jet Fuel.

Natural Attenuation.

Revisado por pares

Recebido em: 24/04/2018

Aprovado em: 18/08/2018.

Abstract

Contaminated site conceptual models are, in general, the first significant result of environmental management. However, it is necessary to understand the complexity of the contaminated compartments to adopt the best remediation alternative of a contaminated site. Subsurface spatial distribution of light non-aqueous phase liquid (LNAPL) usually with high spatial variability and, consequently complex, must be well known. Furthermore, the seasonal water-level fluctuation induces LNAPL trapping and detrapping phenomena and delimits a vertical redistribution zone (smear zone) of contamination, increasing the subsurface complexity. Aiming to solve data gaps left by conventional technique of investigation the laser-induced fluorescence (LIF) technique was used to evaluate the trapping phenomenon in a kerosene-type jet fuel contaminated site situated in the interior of the State of São Paulo. The results of fluorescence tests and water-level measurements in local aquifer show trapped LNAPL in the saturated zone with well-marked boundaries that define the smear zone. Qualitative analysis shows contamination similarity in most of investigated points, with low-intensity fluorescence signal at the shortest response wavelengths, compatible with light hydrocarbons depletion by natural attenuation.

DOI: http://dx.doi.org/10.14295/ras.v32i3.29137

\section{INTRODUÇÃO}

Reconhecidamente, áreas contaminadas geram elevados prejuízos ambientais que abrangem o meio físico e a economia, sendo necessária uma gestão adequada para que possam ser declaradas aptas para sua função natural original ou para uma função específica a ser indicada. Em geral, o gerenciamento de áreas contaminadas busca primeiramente o estabelecimento do modelo conceitual da área, incluindo o meio físico e o cenário de contaminação.

Sendo o modelo conceitual uma simplificação da realidade, verifica- 
se a necessidade do entendimento da complexidade dos meios afetados pela contaminação. Esses meios possuem comportamentos intrínsecos dinâmicos, peculiares e distintos, dificultando o entendimento completo do cenário de contaminação e da definição da melhor alternativa de remediação da área.

Cada vez mais se tem reconhecido que as heterogeneidades geológicas induzem maior variabilidade espacial nas concentrações e no transporte dos contaminantes (DE MARSILY et al., 2005; FORD et al., 2007, FORD et al., 2008). Assim, fica evidente a necessidade de elaboração de abordagens sistemáticas para o desenvolvimento de modelos conceituais representativos de transporte e migração de contaminantes, baseados em ampla distribuição de dados, em escala compatível com as áreas fontes e receptores, e em formas de integração das informações derivadas de dados diretos e indiretos (TREMBLAY et al., 2014).

O vazamento de hidrocarbonetos a partir de dutos ou tanques representa um cenário comum de contaminação. Se envolver volumes suficientemente grandes, o hidrocarboneto liberado em subsuperfície migrará até alcançar a zona saturada, deslocando a água nos poros e se acumulando como LNAPL (Light Non-Aqueous Phase Liquid) na porção superior da zona saturada. No entanto, o nível da água no aquífero flutua em decorrência da alternância de períodos chuvosos com recarga e consequente subida do nível da água, e períodos secos com descarga e queda do nível da água. A flutuação do nivel d'água induz a uma redistribuição vertical do LNAPL à medida que ele migra para porções mais profundas do aquífero com o descenso do nível da água (KEMBLOWSKI e CHIANG, 1990; CHARBENEAU, 2007; JEONG e CHARBENEAU, 2014). Durante o movimento ascendente do nível d'água o LNAPL é retido por força capilar na zona saturada e este fenômeno é comumente conhecido por trapeamento (FARR et al., 1990; LENHARD e PARKER, 1990; KEMBLOWSKI e CHIANG, 1990; MARINELLI e DURNFORD, 1996; CHARBENEAU, 2007). 0 trapeamento do hidrocarboneto restringe a mobilidade do LNAPL, impedindo a sua recuperação por técnicas convencionais de remediação baseadas em bombeamento e tratamento (CHABERNEAU, 2007; PEDE, 2009).

O movimento ascendente e descendente do nível d'água define uma zona de constante redistribuição de LNAPL denominada de smear zone (VAN GEEL e SYKES, 1997; STEFFY et al., 1998; TERAMOTO e CHANG, 2017). A redistribuição do LNAPL deve ser adequadamente avaliada para seleção adequada do programa de remediação (VAN GEEL e SYKES, 1997).

As técnicas convencionais de caracterização das áreas contaminadas deixam lacunas de informação na elaboração de modelos conceituais mais realísticos (NIESSNER et al., 1991), principalmente quanto à delimitação tridimensional da contaminação e determinação da smear zone, pela ausência de um parâmetro confiável de identificação da contaminação e determinado de maneira detaIhada e contínua na direção vertical. 0 preenchimento de tais lacunas pode se dar pelo emprego de técnicas mais avançadas, mais precisas e acuradas, ou de alta resolução (High Resolution Site Characterization - HRSC) (SUTHERSAN et al., 2015), uma vez que essas técnicas se caracterizam, dentre outros fatores, pela determinação in situ de alguns parâmetros, como a fluorescência induzida por laser de espécies oleosas, em intervalos verticais de pequena amplitude e de maneira contínua.
A contaminação por querosene de aviação (QAV) na área de estudo favorece a utilização da técnica de fluorescência induzida por laser (Laser-Induced Fluorescence - LIF). Esta técnica baseia-se no fenômeno da fluorescência de óleos que contenham hidrocarbonetos policíclicos aromáticos (Polycyclic Aromatic Hydrocarbons - PAH) em sua constituição, quando excitadas por luz ultravioleta (TAYLOR e PATTERSON, 1987; NIESSNER et al., 1991; SINFIELD et al., 1999; LÖHMANNSRÖBEN e ROCH, 2000).

Técnicas tradicionais de análise de PAHs em diferentes matrizes despendem tempo desde a coleta de amostras até a análise química, enquanto que ensaios in situ e em tempo real reduzem o tempo de identificação da contaminação (PANNE e NIESSNER, 1993). Espectroscopia por fluorescência apresenta resultados sensíveis para PAHs isolados, mas resultados com baixa resolução para NAPLs (Non-Aqueous Phase Liquid) com multicomposição de PAHs, devido à sobreposição dos espectros de emissão (NIESSNER et al., 1991; PANNE e NIESSNER, 1993), considerando apenas a intensidade e comprimento de onda. Essa limitação pode ser modificada pela inserção de uma terceira dimensão, ou seja, o tempo de emissão de fluorescência, constituindo um diagrama ternário de intensidade, comprimento de onda e tempo (NIESSNER et al., 1991; PANNE e NIESSNER, 1993).

Diferentes NAPLs com diferentes composições quanto aos PAHs possuem diferenças significativas quanto ao rendimento espectral absoluto e ao tempo de decaimento para qualquer comprimento de onda de emissão (CAMAGNI et al., 1991). Interpretações quantitativas da intensidade de fluorescência e qualitativas das respostas de rendimento espectral e tempo de decaimento são a chave da interpretação de ensaios LIF.

O presente estudo analisa o fenômeno de trapeamento de LNAPL, mais precisamente o QAV, por meio da fluorescência induzida por laser dos PAHs constituintes, contemplando a identificação e delimitação vertical do LNAPL presente na smear zone, nas zonas saturada e não saturada.

\section{2. ÁREA DE ESTUDO}

A área de estudo está localizada na região de Campinas-SP, no município de Paulínia (Figura 1), e contida dentro do limite industrial, especificamente na região sudeste da planta industrial, com área de $0,081 \mathrm{~km}^{2}$. A média anual da pluviometria é de $2342 \mathrm{~mm}$, o clima é subtropical úmido com inverno seco e verão quente - Cwa (classificação de Köppen-Geiger). Na região, ocorrem quatro sistemas aquíferos distintos, os Sistemas Aquíferos Cristalino e Serra Geral Intrusivas, de caráter fissural, e Sistemas Aquíferos Tubarão e Cenozoico, sedimentares. Para o estudo, o aquífero de interesse, onde está a contaminação, é o Cenozoico. Esse aquífero, de espessura pouco expressiva (algumas dezenas de metros) é representado por depósitos correlatos à Formação Rio Claro (FERNANDES, 1997), extenso depósitos aluvionares, e caracteriza-se por ser do tipo livre, e poroso intersticial em toda a sua extensão. Esse aquífero apresenta um descompasso de aproximadamente 4 meses entre os máximos valores de precipitação e de ascensão do NA (TERAMOTO, 2007). 0 fluxo geral local da água subterrânea é de nordeste para sudoeste, e a condutividade hidráulica entre $10^{-4}$ e $10^{-6} \mathrm{~m} / \mathrm{s}$ (TERAMOTO, 2007). 
Figura 1 - Localização da área de estudo

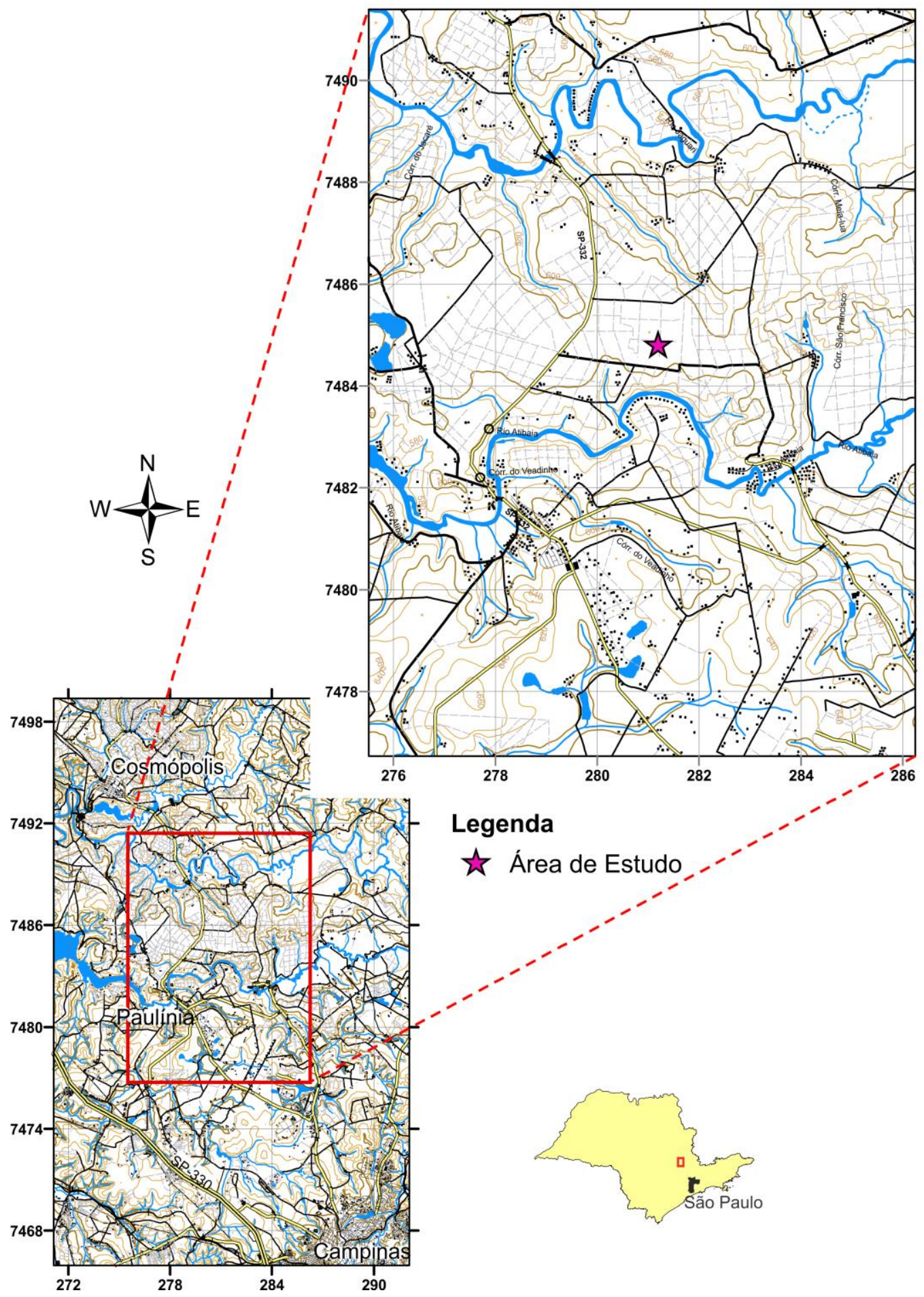

\section{MATERIAIS E MÉTODOS}

Para a observação do fenômeno de trapeamento de LNAPL no aquífero na área estudada foram realizados ensaios de perfilagem contínua por meio da técnica LIF. Para tal, foi utilizando o equipamento UVOST® - Ultra-Violet Optical Screening Tool -, desenvolvido pela Dakota Technologies Inc. Também foi realizado cruzamento dos perfis de fluorescência com dados de nível d’água medidos em poços de monitoramento.

Ao todo, foram realizados 21 ensaios de perfilagem, distribuídos pela área contaminada por QAV. Os pontos selecionados para perfilagem obedeceram três critérios: 1) proximidade de poços de monitoramento ( $1 \mathrm{~m}$ a $19 \mathrm{~m}$ de distância com mediana 2,5 m) com elevada contaminação (fase livre ou concentração dissolvida), conhecida ao longo do período de monitoramento, para registro do Nível d'Água (NA); 2) ampla distribuição pela área contaminada e 3) que permitisse distinção entre possíveis fontes de contaminação. A distância entre os pontos de perfilagem e poços de monitoramento não interferiu na correlação entre os resultados de LIF e os dados de NA, uma vez que o comportamento da potenciometria na área não apresenta grande variação. 


\subsection{Ensaios de Perfilagem por Fluorescência Induzida por Laser}

O ensaio de perfilagem pelo método de cravação contínua direct push consiste na inserção direta e contínua da ferramenta no substrato, desejavelmente com velocidade constante compatível com a técnica utilizada. Para a perfilagem com o UVOST® é requerida velocidade máxima de penetração de $2 \mathrm{~cm} / \mathrm{s}$. Velocidades superiores podem provocar desencontro entre resposta de fluorescência e o sistema óptico, induzindo leituras de menor intensidade ou até mesmo falso negativo.
O UVOST ${ }^{\circledR}$ consiste basicamente em quatro módulos: a) fonte de emissão (laser do tipo excimer), que gera luz ultravioleta (UV) com comprimento de onda de $308 \mathrm{~nm}$; b) módulo de transmissão de luz, que consiste em cabo flexível dotado de duas fibras ópticas, uma para condução da luz UV e outra para recepção de fluorescência, e uma ponteira de aço inox reforçado com sistema de redução de choque, dotado de conjunto óptico (espelho parabólico e janela de safira); c) módulo de conversão de sinal, ou tubo fotomultiplicador e osciloscópio, para transformação do sinal luminoso em sinal elétrico e para separação da luz fluorescente em quatro faixas de comprimentos de onda distintos: $350 \mathrm{~nm}, 400 \mathrm{~nm}, 450 \mathrm{~nm}$ e $500 \mathrm{~nm}$; d) módulo de interpretação de informação - computador (Figura 2).

Figura 2 - Representação esquemática do sistema direct push utilizado para a perfilagem e do equipamento (módulos) UVOST $\AA$, com detalhe em foto da unidade utilizada

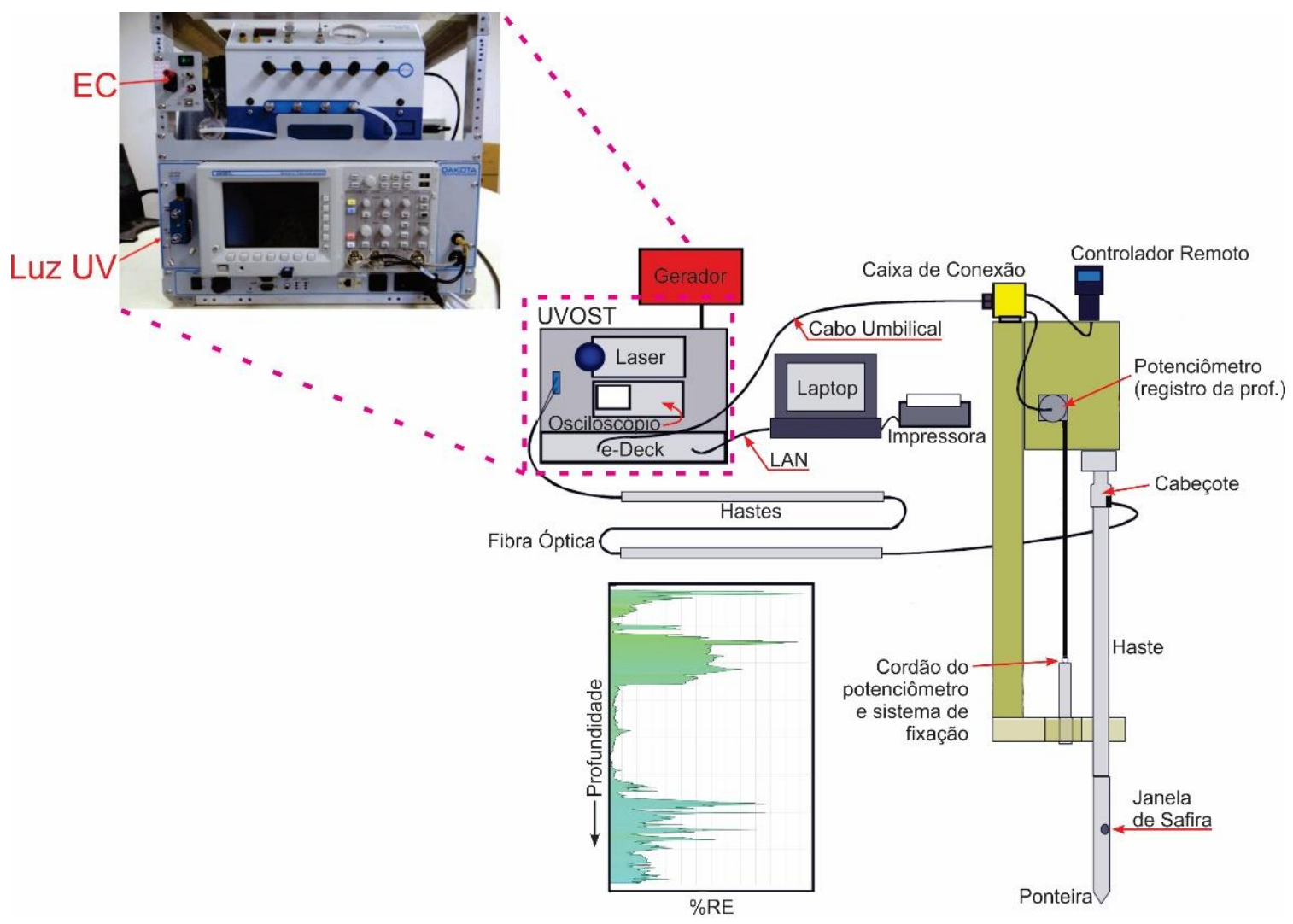

Fonte: Adaptado de ST. GERMAIN (2012)

A perfilagem contínua com o UVOST $\circledast$ fornece resultados, em tempo real, da intensidade de fluorescência como resposta à incidência de energia gerada por laser nas moléculas de PAH. A luz UV, quando atinge o meio com LNAPL contendo PAH em sua composição, induz o fenômeno da fluorescência (absorção - mudança de estado de energia - emissão de fluorescência - Figura 3). Esta fluorescência é, então, captada e enviada de volta pela fibra óptica até o módulo de conversão, para então ser interpretado como intensidade de fluorescência pelo software conectado com o equipamento. Esta intensidade de fluorescência é registrada de acordo com o avanço da cravação da ferramenta no subsolo, gerando uma curva intensidade de fluorescência versus profundidade para cada ponto avaliado ( $\mathrm{Fi}$ gura 4). 
Figura 3 - Diagrama de Energia de Jablonski para a fluorescência e fluorescência retardada

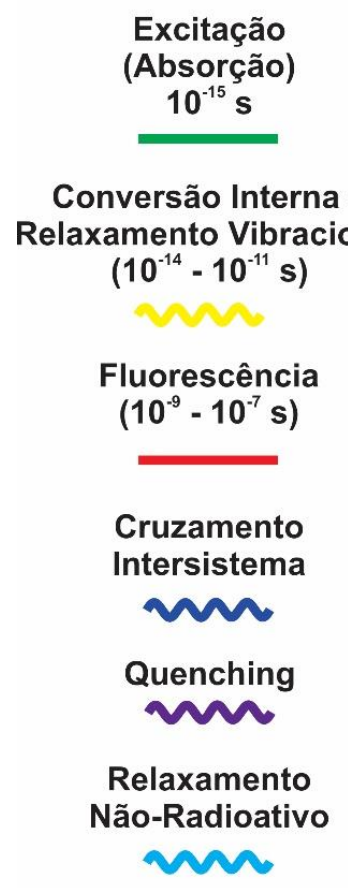

Excitação

(Absorção)

$10^{-15} \mathrm{~s}$

Conversão Interna e

$\left(10^{-14}-10^{-11} \mathrm{~s}\right)$

Fluorescência

$\left(10^{-9}-10^{-7} s\right)$

Quenching

Relaxamento

M

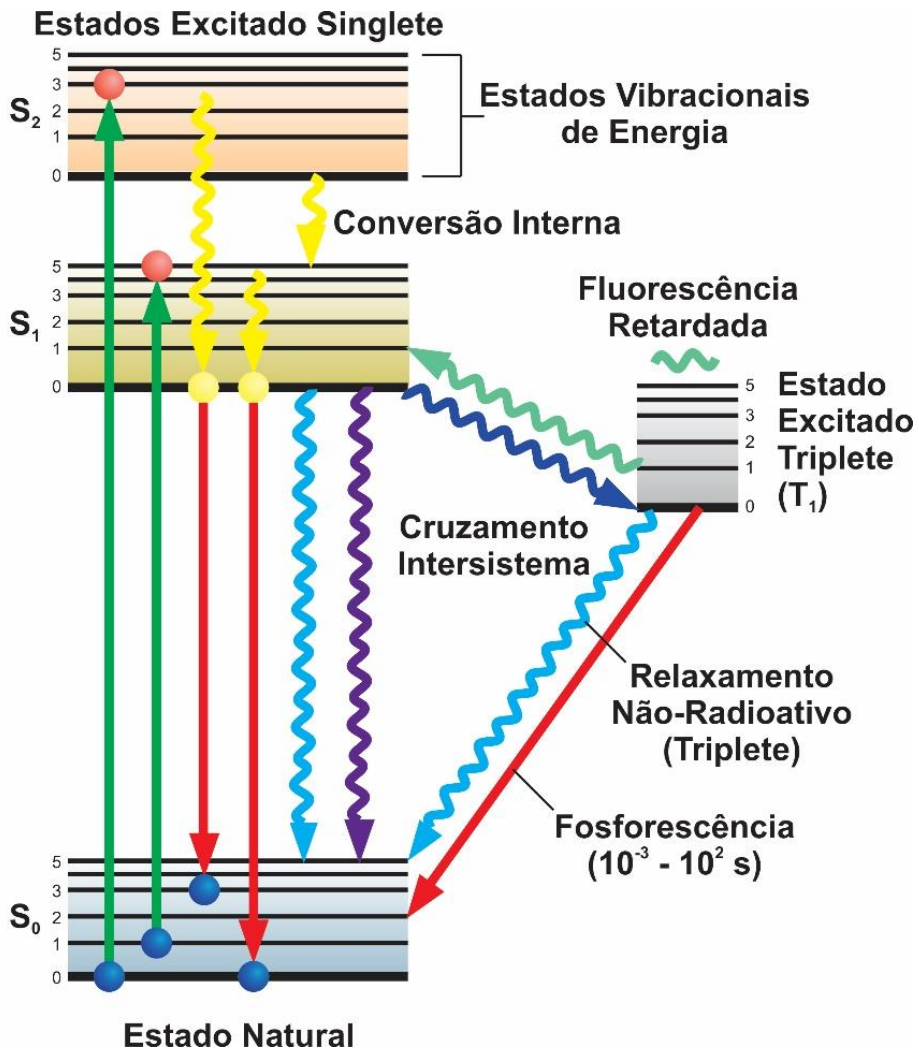

Fonte: Adaptado de Perez (2011)

$\mathrm{Na}$ fluorescência os elétrons no estado natural $\mathrm{S}_{0}$ absorvem energia, saltando para os estados vibracionais $\mathrm{S}_{1}$ e $\mathrm{S}_{2}$ de mais alta energia, e emitem fluorescência, ao retornar para o estado natural So.

Por ser um fenômeno óptico, a fluorescência não é limitada pela presença de água no meio, tornando o UVOST $\AA$ eficiente nos meios saturados e não saturados. Sinfield et al. (1999) afirmam que as propriedades das partículas do substrato não produzem efeitos mensuráveis na assinatura da fluorescência (natureza e magnitude), uma vez que esta é função da assinatura do contaminante. Entretanto, para esses mesmos autores, alguns aspectos podem interferir na identificação da fluorescência, principalmente o tamanho das partículas do meio, sendo a resposta do LIF inversamente proporcional à área superficial da matriz e, secundariamente, à mineralogia, à cor e ao teor de matéria orgânica.

A perfilagem fornece informações qualitativas quanto ao tipo de óleo presente com base no padrão da resposta de fluorescência. 0 aspecto qualitativo da fluorescência é registrado em gráficos de formas de onda com multicomprimentos de onda (Multi-Wavelength Waveform, ou simplesmente waveform) (Figura 5), os quais representam as variáveis rendimento espectral e tempo de decaimento. Esses gráficos são gerados a partir de gráficos 3D em que são indicados os parâmetros comprimento de onda, tempo de decaimento e intensidade, designados como WTIM (Wavelength-Time-Intensity Matrix). Esses parâmetros são dependentes do comprimento da cadeia policíclica dos hidrocarbonetos presentes. Cada composto químico emite fluorescência com uma WTIM característica, permitindo a distinção entre produtos de acordo com as formas dos quatro picos das waveforms.

Esses perfis também fornecem informações semiquantitativas quanto ao conteúdo de óleo presente: quanto maior a quantidade de óleo presente, mas intensa é a resposta de fluorescência (Figura 5). As intensidades de resposta são comparadas com um padrão de calibração (Reference Emitter - RE).

A perfilagem dos 21 pontos LIF na área contaminada por QAV foi realizada em três etapas, a pioneira realizada em junho de 2013 (ponto T-01), a segunda realizada em setembro de 2015 (pontos S01 a S-06), e a última realizada em março de 2017 (pontos S-07 a S-14 e S-20 a S-25). 
Figura 4 - Perfil de Intensidade de Fluorescência, mostrando os aspectos semiquantitativo com a Curva de Sinal de Fluorescência qualitativo com as Waveforms, além do registro da velocidade de penetração da ponteira Indicação
de Profundidade

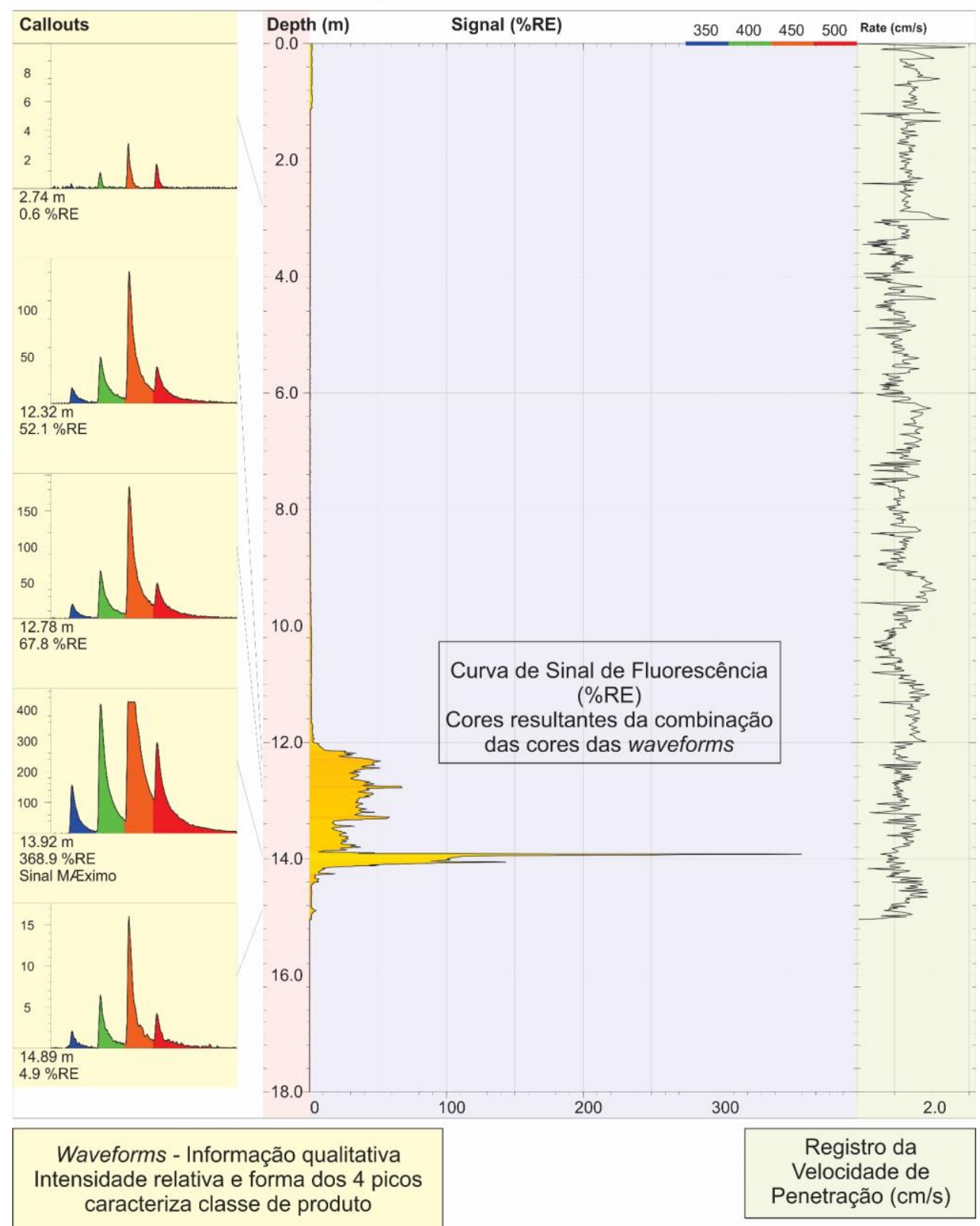




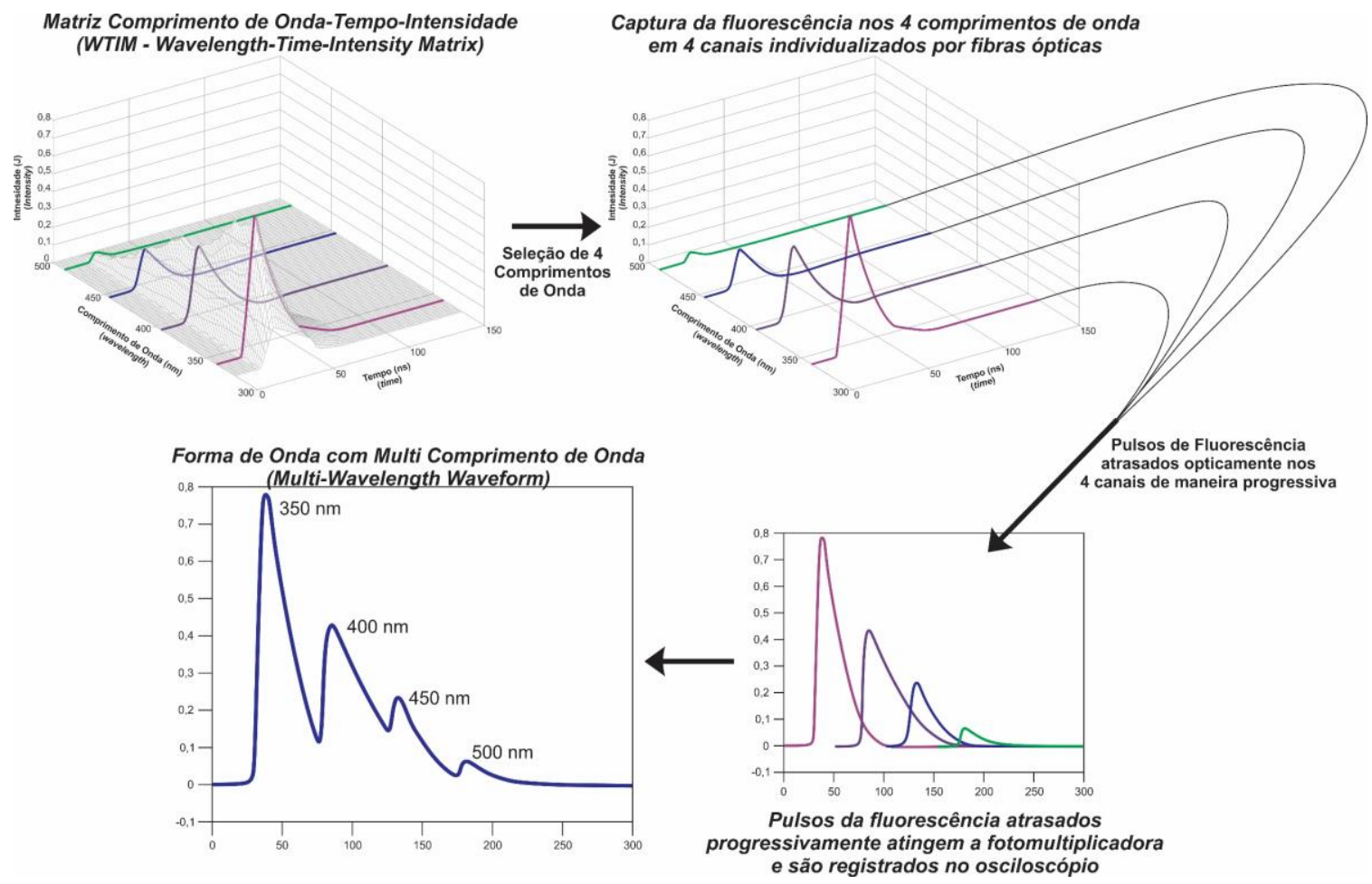

Fonte: Adaptado de St. Germain (2012)

\subsection{Monitoramento do Nível d'Água Local}

Na área de estudo existem 107 poços de monitoramento, sendo 84 destes situados dentro da área fonte (fase residual), com levantamento planialtimétrico georreferenciado segundo projeção UTM (Universal Transversa de Mercator) Zona 23S, datum SIRGAS2000. Estes 84 poços possuem séries históricas de NA, de espessura de LNAPL e de monitoramento analítico, dentre os quais, 20 foram selecionados pelo longo período de monitoramento dos parâmetros de interesse.

As leituras recentes de NA foram realizadas com medidor portátil do tipo interface, da marca Solinst, modelo $122 \mathrm{M}$, tendo como referenciais a boca do poço e a superfície do terreno. Nos poços com identificação de fase livre sobrenadante foram medidas as espessuras. Todas as medidas tomadas de profundidade de NA e espessura de LNAPL foram inseridas nos perfis de fluorescência.

O monitoramento do NA ocorreu em 4 etapas, as três primeiras coincidentes com as etapas de perfilagem LIF (junho/2013, setembro/2015 e março/2017), e a última realizada em dezembro/2017.

\section{RESULTADOS E DISCUSSÃO}

A partir dos dados de profundidade do NA de cada ponto perfilado pela técnica LIF foi possível identificar o comportamento da contaminação por LNAPL na área de interesse. Os resultados dos ensaios LIF indicam trapeamento de LNAPL na porção saturada do meio.

O fenômeno do trapeamento de QAV na área de estudo foi descrito por Pede (2009), Teramoto (2015) e Teramoto e Chang (2017). Pelos ensaios LIF foi possível identificar, em detalhe, os limites superior e inferior da zona contaminada por QAV, acima e abaixo do NA (Figura 6).

A perfilagem pioneira no ponto T-01, realizada em junho de 2013, teve por objetivo testar a ferramenta e a técnica em uma área contaminada já conhecida (PEDE, 2009; BORDIGNON, 2014; TERAMOTO, 2015; TERAMOTO e CHANG, 2017). O resultado obtido (Figura $6 a$ ) demonstrou que o limite superior do horizonte contaminado $(10,10 \mathrm{~m})$ coincidiu com a linha do NA $(9,99 \mathrm{~m})$ medido no poço PM-86 adjacente, indicando trapeamento, como descrito por Pede (2009). Na campanha de setembro de 2015, foi perfilado o ponto S-05 (Figura 6b), muito próximo ao ponto T-01, sendo observado comportamento semelhante, com NA em 11,75 m. Em dezembro de 2017, foi medido o NA no poço PM-86 em 10,08 m, revelando elevação de 1,67 m na superfície potenciométrica, com LNAPL trapeado aproximadamente $2 \mathrm{~m}$ abaixo do NA.

No ponto S-02 (Figura 6c), a situação foi semelhante ao ponto S-05. 0 ponto S-04 (Figura 6d) revelou sinal intenso de fluorescência (máximo de 157,9\%RE) entre as profundidades de 11,2 m e 14,6 m, e NA em 12,05 m, indicando LNAPL nas formas livre acima e residual abaixo do NA, e corroborado pela medição de 0,204 $\mathrm{m}$ de fase livre no poço PM-100A localizado próximo.

A campanha realizada em março de 2017 apresentou resultados semelhantes da contaminação, em cenário hidrogeológico de NA mais raso após intensa recarga. 0 ponto S-07 (Figura 6e) apresentou o maior sinal de fluorescência (368,9\%RE) dentre todos os pontos avaliados nas três etapas de perfilagem. Durante a perfilagem, 
o NA medido em 9,62 m revelou LNAPL trapeado 2,4 m abaixo. 0 NA medido em 12,22 m no poço PM-115A, em setembro de 2015, indicou 0,2 m de LNAPL na forma livre móvel acima do NA, corroborado pela medição de $0,052 \mathrm{~m}$ de fase livre no poço. Como esperado, não foi observada fase livre no poço PM-115A em 2017, com NA 2,4 m mais raso.

No ponto S-09 (Figura 6f) foi verificada fluorescência entre 11,4 m e 13,6 m, com intensidade máxima de 310,4\%RE e NA em 9,93 m. Considerando o cenário de 2015, embora no poço PM-84 adjacente não tenha sido identificada fase livre, o NA em 12,15 m sugere LNAPL na forma livre naquele intervalo.

A perfilagem realizada no ponto S-23 (Figura 6g), próximo ao limite leste da área fonte, revelou espessura de 2,2 m de LNAPL distribuído no meio (9,6 m a 11,8 m), com intensidade máxima de 29\%RE e NA em 8,8 m, indicando trapeamento de LNAPL no aquífero. Em dezembro de 2017 foi medido o NA em 9,85 m no poço PM-95, o que indica um descenso de 1,05 $\mathrm{m}$ em nove meses, deixando pequena parcela de LNAPL acima do NA passível de mobilidade. A situação identificada no ponto S-24 (Figura 6h) é semelhante à dos demais.

Figura 6 - Perfis de Intensidade de Fluorescência com a profundidade nos pontos T-01, S-02, S-04, S-05, S-07, S-09, S-23 e S-24. Indicações das profundidades de NA medidos em 04 períodos distintos (jun/2013, set/2015, mar/17 e dez/2017)
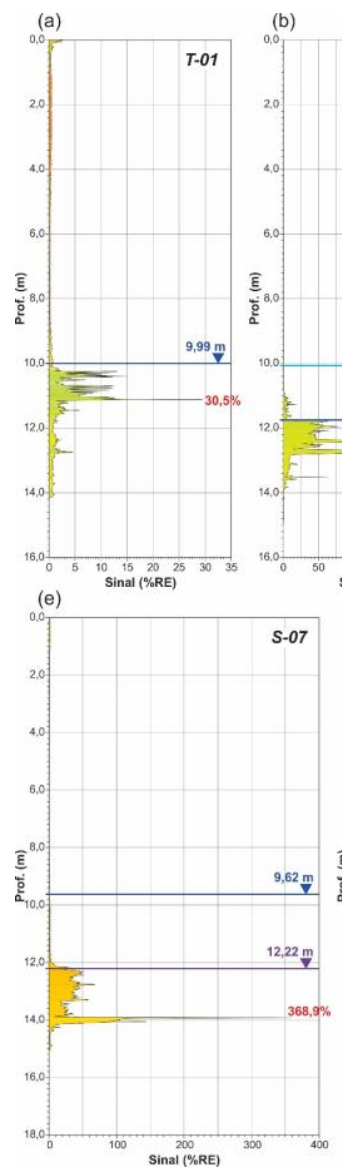
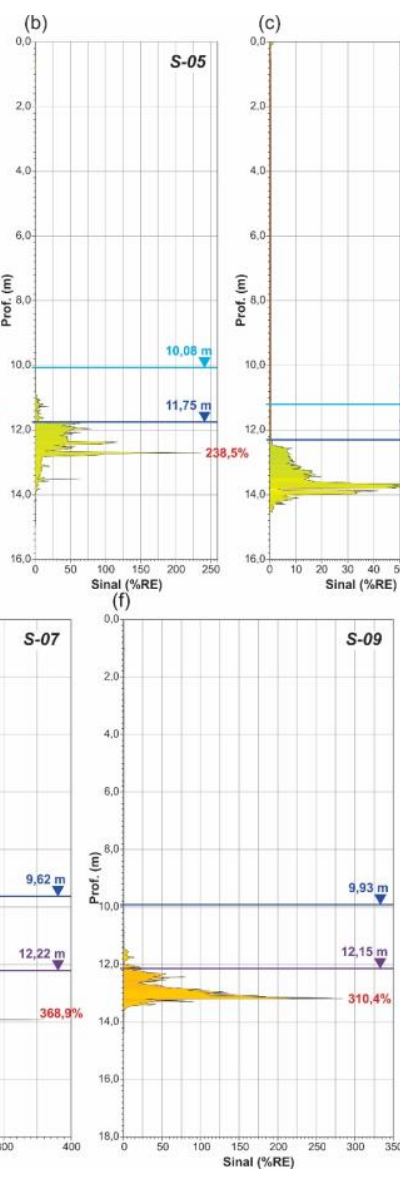
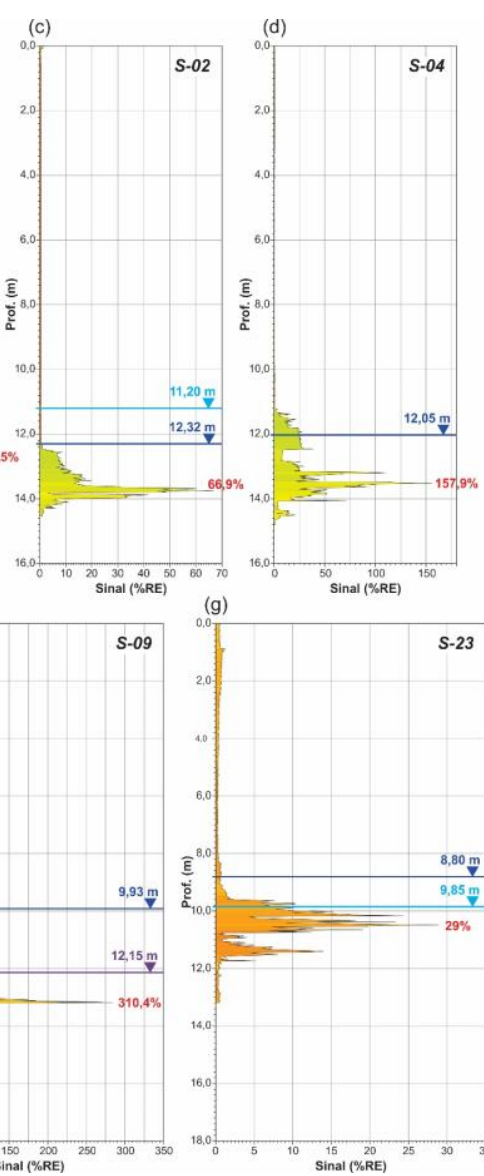
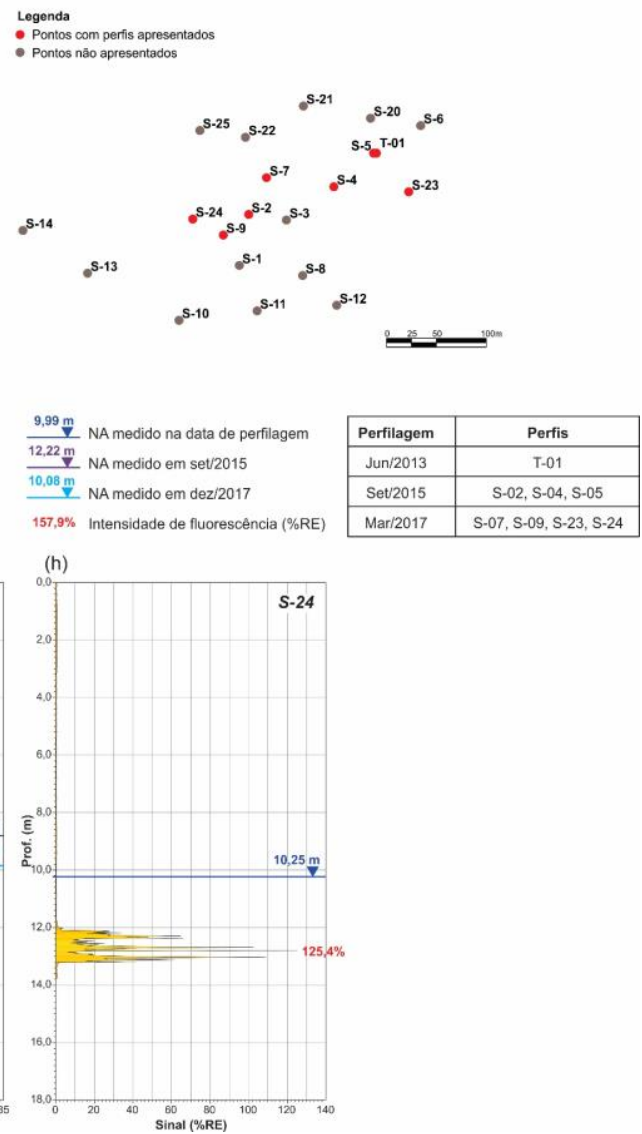

Para a interpretação qualitativa, alguns aspectos devem ser considerados. 1) O conhecimento do modelo conceitual da contaminação auxilia na distinção do LNAPL; estudos anteriores revelam que o contaminante na área é querosene de aviação. 2) Processos de degradação por atenuação natural depletam o LNAPL em hidrocarbonetos de cadeias policíclicas curtas por transferência difusiva contínua de massa para a água, acarretando supressão significativa do sinal de fluorescência, principalmente nos comprimentos de onda de $350 \mathrm{~nm}$ e $400 \mathrm{~nm}$. 3) Os processos de transferência de massa de contaminante não são regulares em toda a área fonte. 4) Elevada intensidade de fluorescência pode alterar a forma de algum pico (comprimento de onda específico). 5) É necessário avaliar as intensidades relativas entre os quatro picos e o tempo de decaimento da fluorescência para cada comprimento de onda.
Os padrões das waveforms nos pontos perfilados (Figura 7 a-h) indicam similaridades dos produtos encontrados na maioria dos pontos. De maneira geral, são observados três padrões temporais quanto às respostas de fluorescência nos comprimentos de onda de $350 \mathrm{~nm}$ e $400 \mathrm{~nm}$. É observada diminuição das intensidades das respostas nestes comprimentos de onda no ponto T-01 em 2013 (Figura 7a), passando por 2015 (pontos S-05, S-02 e S-04, respectivamente, Figuras 7b a 7d) até 2017 (pontos S-07, S-09, S-23 e S24 , respectivamente, Figuras $7 \mathrm{e}$ a $7 \mathrm{~h}$ ). Para todos os casos, a resposta de fluorescência no comprimento de onda de $500 \mathrm{~nm}$ foi baixa, como esperado para o QAV, composto essencialmente por hidrocarbonetos leves. 
Figura 7 - Padrões das waveforms dos pontos T-01, S-02, S-04, S-05, S-07, S-09, S-23 e S-24, além de uma amostra de QAV recém-processado e outra amostra de QAV recuperado em sistema de remediação instalado na área
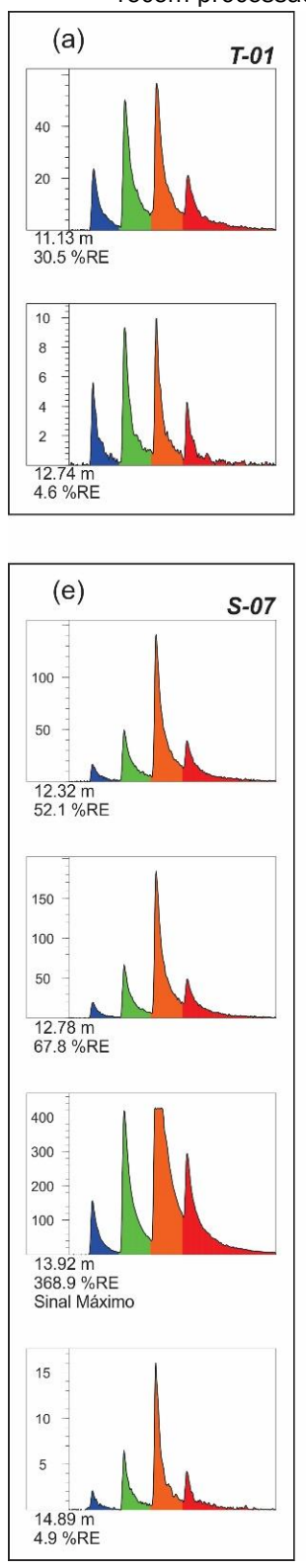
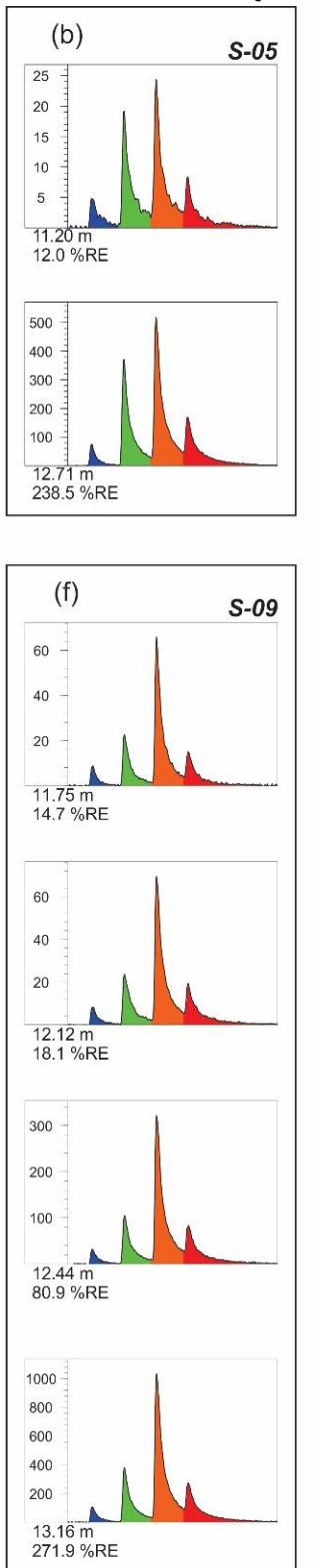
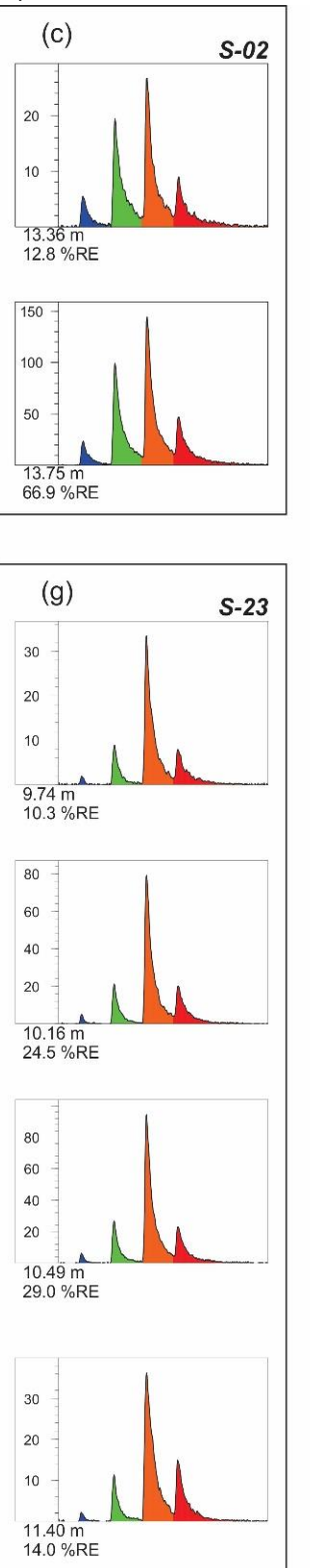
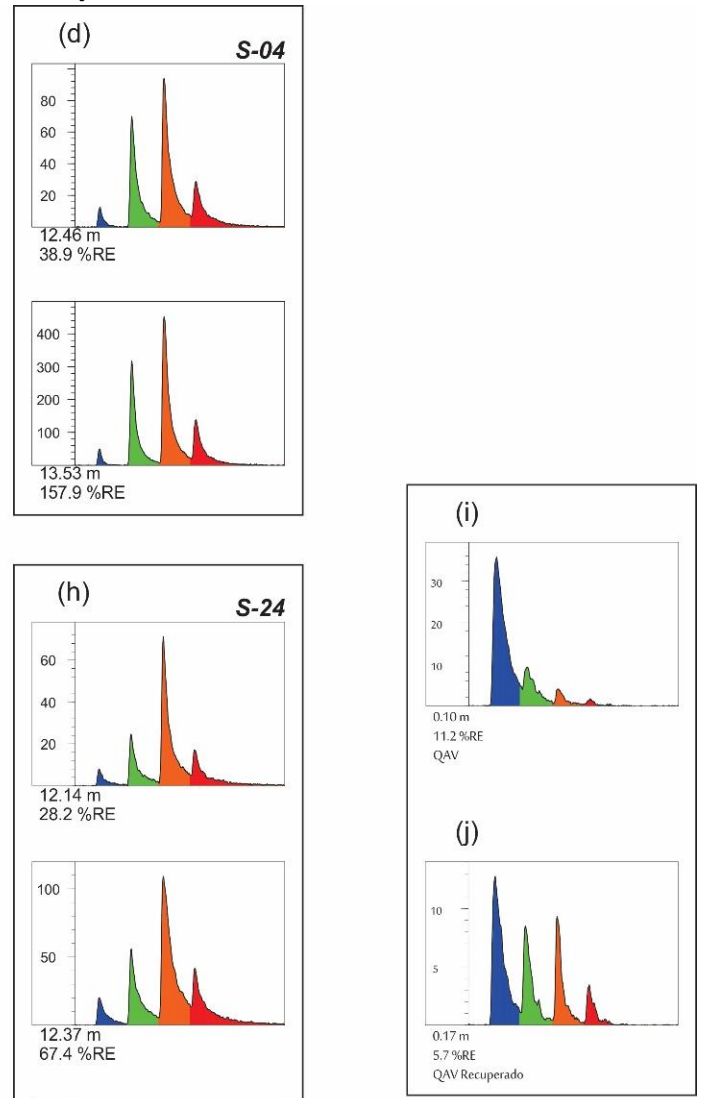

Em 2013 foi realizado um teste de bancada prévio ao teste de campo, utilizando duas amostras de QAV oriundas da área de estudo. Uma amostra de QAV recém-processado foi testada com o UVOST $\AA$, resultando em uma waveform característica do produto (Figura 7i), com pico proeminente em $350 \mathrm{~nm}$. Outra amostra de QAV coletada no sistema de remediação também foi testada (Figura $7 \mathrm{j})$, resultando em uma waveform com relação diferente entre comprimentos de onda, com menor resposta em $350 \mathrm{~nm}$ quando comparado aos demais, resultante da depleção em hidrocarbonetos policíclicos mais leves por transferência de massa para a água subterrânea.

\section{CONCLUSÕES}

A técnica de fluorescência induzida por laser mostrou-se eficiente no estudo do fenômeno do trapeamento de contaminantes na zona saturada. Os resultados obtidos permitiram delimitar com clareza os limites da zona de redistribuição (smear zone) da contaminação

por hidrocarbonetos, causada pela flutuação sazonal do nível freático do aquífero local, o que permitiu a identificação do intervalo de contaminante nas zonas saturada e não saturada. Esses resultados são fundamentais para o planejamento de sistemas de remediação e do regime ou janela de operação que considere a sazonalidade de trapeamento e destrapeamento de contaminante.

\section{AGRADECIMENTOS}

Os autores agradecem à PETROBRAS pela cessão da área, ao LE$\mathrm{BAC} /$ Unesp pelo apoio técnico e infraestrutura e à CAPES pelo financiamento da bolsa de doutorado (Elias Isler).

\section{REFERÊNCIAS}

BORDIGNON, R. Caracterização Isotópica do $\mathrm{CO}_{2}$ dissolvido em águas subterrâneas no munícipio de Paulínia, SP. Dissertação 
(Mestrado em Geociências e Meio Ambiente). Instituto de Geociências e Ciências Exatas, Universidade Estadual Paulista. Rio Claro. 106 f. 2014.

CAMAGNI, P. et al. Fluorescence response of mineral oils: spectral yield vs absorption and decay time. Applied Optics, v. 30, n. 1, p. 2635, 1991.

CHARBENEAU, R.J. LNAPL distribution and recovery model (LDRM). API Publication Number 4760, American Petroleum Institute, Washington D.C., 2007.

DE MARSILY, G. et al. Dealing with spatial heterogeneity. Hydrogeology Journal, v. 13, n. 1, p. 161-183, 2005.

FARR, A. M. et al. Volume estimation of light nonaqueous phase liquids in porous media. Groundwater, v. 28, n. 1, p. 48-56, 1990.

FERnANDES, A. J. Tectônica Cenozóica na Porção Media da Bacia do rio Piracicaba e sua Aplicação à Hidrogeologia, Tese (Doutoramento em Recursos Minerais e Hidrogeologia). Instituto de Geociências, Universidade de São Paulo. São Paulo, 244 F, 1997.

FORD, R. G. et al. Monitored natural attenuation of inorganic contaminants in ground water: Technical basis for assessment. Cincinati: US Environmental Protection Agency, National Risk Management Research Laboratory, 2007.

FORD, R. G. et al. Site characterization to support use of monitored natural attenuation for remediation of inorganic contaminants in ground water. Cincinnati: US Environmental Protection Agency, National Risk Management Research Laboratory, 2008.

JEONG, J.; CHARBENEAU, R. J. An analytical model for predicting LNAPL distribution and recovery from multi-layered soils. Journal of Contaminant Hydrology, v. 156, p. 52-61, 2014.

KEMBLOWSKI, M. W.; CHIANG, C. Y. Hydrocarbon thickness fluctuations in monitoring wells. Groundwater, v. 28, n. 2, p. 244-252, 1990.

LENHARD, R. J.; PARKER, J. C. Estimation of free hydrocarbon volume from fluid levels in monitoring wells. Groundwater, v. 28, n. 1, p. 57-67, 1990.

LÖHMANNSRÖBEN, H.-G.; ROCH, T. In situ laser-induced fluorescence (LIF) analysis of petroleum product-contaminated soil samples. Journal of Environmental Monitoring, v. 2, n. 1, p. 17-22, 2000.

MARINELLI, F.; DURNFORD, D. S. LNAPL thickness in monitoring wells considering hysteresis and entrapment. Groundwater, v. 34, n. 3, p. 405-414, 1996.

NIESSNER, R. et al. Fibre-optic sensor for the determination of polynuclear aromatic hydrocarbons with time-resolved, laser-induced fluorescence. Analytica Chimica Acta, v. 255, n. 2, p. 231-243, 1991.

PANNE, U.; NIESSNER, R. A fiber-optical sensor for polynuclear aro- matic hydrocarbons based on multidimensional fluorescence. Sensors and Actuators B: Chemical, v. 13, n. 1-3, p. 288-292, 1993.

PEDE, M. A. Z. Flutuação do lençol freático e sua implicação na recuperação de hidrocarbonetos: um estudo de caso. Tese (Doutorado em Geociências e Meio Ambiente). Instituto de Geociências e Ciências Exatas, Universidade Estadual Paulista. Rio Claro, 126 f. 2009.

PEREZ, E. Couches moléculaires sur silicium pour la détection sélective de protéines. Tese (Doutorado) - Ecole Polytechnique Palaiseau, 173 f. 2011.

SINFIELD, J. V. et al. Effects of soils on laser induced fluorescence of BTX contaminated pore waters. Journal of Geotechnical and Geoenvironmental Engineering, v. 125, n. 12, p. 1072-1077, 1999.

ST. GERMAIN, R. UVOST Training: LIF Data Interpretation and UVOST System Review. Fargo: Dakota Technologies, Inc, 2012. 162 slides, cor.

STEFFY, D. A. et al. A. Numerical simulations and long-column tests of LNAPL displacement and trapping by a fluctuating water table. Journal of Soil Contamination, v. 7, n. 3, p. 325-356, 1998.

SUTHERSAN, S. et al. The new ROI: return on investigations by utilizing smart characterization methods. Ground. Monit. \& Remed., v. 35, n. 3, p. 25-33, 2015.

TAYLOR, T. A.; PATTERSON, H. H. Excitation resolved synchronous fluorescence analysis of aromatic compounds and fuel oil. Analytical Chemistry, v. 59, n. 17, p. 2180-2187, 1987.

TERAMOTO, E. H. Caracterização hidrogeológica e simulação numérica de fluxo em uma região situada no Distrito Industrial de Paulínia $(\mathrm{SP})$. Dissertação (Mestrado em Geociências e Meio Ambiente). Instituto de Geociências e Ciências Exatas, Universidade Estadual Paulista. Rio Claro, 96 f. 2007.

TERAMOTO, E. H. Estudo da efetividade da atenuação natural de compostos BTEX em área contaminada por querosene de aviação. Tese (Doutoramento em Geociências e Meio Ambiente). Instituto de Geociências e Ciências Exatas, Universidade Estadual Paulista. Rio Claro, 215 f. 2015.

TERAMOTO, E. H.; CHANG, H. K. Field data and numerical simulation of BTEX concentration trends under water table fluctuations: Example of a jet fuel-contaminated site in Brazil. Journal of Contam. Hydrology, v. 198, p. 37-47, 2017.

TREMBLAY, L. et al. Conceptual model of leachate migration in a granular aquifer derived from the integration of multi-source characterization data (St-Lambert, Canada). Hydrogeology Journal, v. 22, n. 3, p. 587-608, 2014.

VAN GEEL, P. J.; SYKES, J. F. The importance of fluid entrapment, saturation hysteresis and residual saturations on the distribution of a lighter-than-water non-aqueous phase liquid in a variably saturated sand medium. Journal of Contaminant Hydrology, v. 25, n. 34, p. 249-270, 1997. 